引用格式: 蒋依依, 汲忠娟, 谢婷. 居民与社区关系视角下台湾居民对大陆赴台旅游支持度研究 [J]. 资源科学, 2019, 41(5): 943954. [Jiang Y Y, Ji Z J, Xie T. Attitude of Taiwan residents towards tourism from China's mainland[J]. Resources Science, 2019, 41 (5): 943-954.] DOI: 10.18402/resci.2019.05.11

\title{
居民与社区关系视角下台湾居民对大陆赴台 旅游支持度研究
}

\author{
蒋依依 ${ }^{1,2}$, 汲忠娟 ${ }^{3}$, 谢 婷 $^{4}$ \\ (1. 北京体育大学体育休闲与旅游学院, 北京 100084 ; \\ 2. 中国旅游研究院国际旅游研究所, 北京 100005 ; \\ 3. 中山大学旅游学院, 珠海 519082 ; \\ 4. 北京石油化工学院旅游与会展系, 北京 102617)
}

\begin{abstract}
摘 要: 为揭示社区居民旅游支持度及影响机理, 本文基于社会交换理论,构建了社区依恋、社区关注、旅游获 益感知、旅游成本感知和旅游支持度 5 个要素组成的概念模型, 采用现场问卷调查法就台湾地区居民对大陆游客赴 台旅游的支持度进行了调研, 并运用结构方程模型对 341 份有效问卷进行了数据分析。研究发现: 台湾地区居民的 获益感知和成本感知对大陆赴台旅游的支持度会产生直接的正向影响; 社区关注不仅直接正向影响居民旅游支持 度, 同时还会通过获益感知间接正向影响居民旅游支持度; 社区依恋会通过获益感知间接负向影响支持度; 社区依 恋和社区关注之间的交互作用并没有对支持度产生影响。台湾居民与社区的关系对旅游地居民的旅游支持度既 有直接影响也有间接影响, 旅游地相关政策制定需重视维护居民与社区关系, 并积极提高居民在旅游发展中的参 与度,通过保障和协调居民一社区关系提高居民对旅游发展的支持度。
\end{abstract}

关键词: 居民一社区关系; 旅游支持度; 影响因素; 社区依恋; 社区关注

DOI :10.18402/resci.2019.05.11

\section{1 引言}

居民在旅游地发展中承担着多重社会角色, 既 是旅游开发活动的影响者和参与者, 也是利益相关 者的主体之一[1-3]。因此, 居民是旅游人地关系研究的 重要切人点 ${ }^{[4-6]}$ 。作为旅游发展影响的直接受众, 居民 对旅游发展的感知、态度与其在旅游发展中的各种 行为倾向是协调一致的 ${ }^{[7]}$ 。感知是居民对居住空间 的物质和社会文化环境改变的主观认知; 态度是居 民对旅游地变化的主动响应; 行为是态度的外显 ${ }^{[8]}$ 。 所以, 居民正负面感知与相应的支持度成为旅游地
旅游可持续发展的先决条件 ${ }^{[9]}$ 。居民感知与态度之 间往往具有较强的相关性 ${ }^{[10,11]}$, 其关系的强度会受到 各种异质性因素的影响 ${ }^{[12]}$, 其中居民一社区关系被认 为是重要的影响因素 ${ }^{[13]}$ 。因此, 辨析居民感知与态度 之间的相互关系, 特别是对其产生影响的相关因素, 有助于针对性地提升居民旅游发展支持度。

自 2008 年 7 月正式开放大陆居民赴台旅游以 来, 大陆逐渐成为台湾重要旅游客源市场。台湾地 区统计数据显示, 大陆游客从 2008 年的 32.9 万人次 增长至 2015 年的 418.4 万人次, 其间 2013 年大陆取

收稿日期: 2018-07-22, 修订日期: 2018-11-14

基金项目: 国家 “万人计划” 青年拔尖人才课题; 国家旅游局科研立项重点项目(14TACK009); 国家旅游局旅游业青年专家培养计划课题 (TYEPT201404)。

作者简介:蒋依依,女,贵州贵阳人,博士, 教授,博士生导师,研究方向为旅游市场、生态旅游等。E-mail: yiyijiangpku@126.com

通讯作者:谢婷, E-mail:shur_shur@126.com

http://www.resci.cn 
代日本成为台湾地区最大客源地, 2015 年大陆游客 占赴台旅游人数的 $40.1 \%{ }^{[14]}$ 。但因台湾地区政治生 态过于放大部分民众的声音, 使少数反对意见影响 到包括旅游业在内的经济政策出台,进而使两岸经 贸协商成果未能转化为现实市场 ${ }^{[15]}$ 。受多种因素影 响, 2016、2017年大陆游客赴台数量持续减少。从 现有研究来看, 学者们通过详尽测算, 认为较快增 长的大陆游客对台湾社会经济增长与两岸文化交 流作出较大贡献 ${ }^{[16]}$, 同时也理性分析了游客增长导 致的对自然环境压力增大、游客拥挤的挤出效应等 问题 ${ }^{[17]}$ 。基于此, 两岸学者对台湾居民对大陆游客 赴台的感知与态度进行了研究, 但以描述性统计 研究为主, 主要关注对大陆游客赴台旅游影响的 感知 ${ }^{[18-20]}$, 居民态度研究十分有限, 探索其感知、态 度之间关系及其影响因素的研究更为贵乏; 且主要 集中在 2015 年之前, 缺乏对于大陆游客赴台人次下 降后居民的旅游支持度研究。对于台湾地区而言, 居民的支持度不仅会影响大陆游客的认知与满意 度, 而且可能在很大程度上左右大陆与台湾地区之 间的旅游市场政策,进而影响两岸之间的正常往来 与交流, 因此深人了解台湾居民对于大陆游客赴台 旅游支持度的研究不仅具有学术价值还有重要的 应用价值。

本文在相关研究基础上 ${ }^{[2,22]}$, 以台湾地区居民 为研究对象, 基于社区依恋、社区关注理论刻画居 民一社区关系, 依据社会交换理论将居民感知划分 为获益感知与成本感知, 构建居民一社区关系、居 民感知与旅游支持度之间的关系模型, 并探索分析 居民一社区关系对居民支持度的直接影响, 以及居 民一社区关系变量的相互作用, 以深人剖析台湾居 民对大陆游客赴台旅游支持度的影响机理, 掌握大 陆赴台旅游过程中主客互动关系, 为旅游市场政策 制度提供针对性政策与措施, 促进大陆赴台旅游的 可持续发展。

\section{2 文献回顾与研究假设}

\section{1 居民感知}

居民对旅游发展所持态度受到感知的影响 ${ }^{[23,24]}$ 在旅游地居民感知和态度研究中, 社会交换理论应 用较广 ${ }^{[25,26]}$ 。该理论认为, 人类的社会行为是交换 过程的产物, 人们交换的目的就是实现成本最小化
和利益最大化 ${ }^{[27]}$ 。依据社会交换理论,感知可划分 为获益感知和成本感知 ${ }^{[28]}$ 。相关研究表明, 居民对 旅游发展的支持度取决于在当地旅游过程中取得 的收益与付出的成本 ${ }^{[29,30]}$ 。当居民感知到获益大于 成本时,他们对旅游业的发展将会持积极支持的态 度, 反之则会持消极抵制的态度 ${ }^{[31-33]}$ 。Vargassánchez 等 ${ }^{[34}$ 和 Nunkoo 等 ${ }^{[30]}$ 等通过回归分析证实旅游获益 感知可以准确预测居民旅游支持度。基于此, 本文 提出以下假设：

$\mathrm{H} 1$ : 旅游获益感知对旅游支持度具有影响;

$\mathrm{H} 2$ : 旅游成本感知对旅游支持度具有影响。

\section{2 居民一社区关系}

社区 (Community) 概念由德国社会学家 Toen$n i e s^{[35]}$ 于 1887 年首次提出, 指存在于传统社会中的 人与人之间关系密切, 具有血缘、情感的共同利益 体。居民一社区关系, 即居民和与他们之间有着共 同的意识、利益、社会交往较为密切的地理区域之 间的联系。相关研究通常选择社区依恋和社区关 注分别测评居民对社区的情感和行为 ${ }^{[11]}$ 。

\subsection{1 社区依恋 (Community Attachment,CA)}

社区依恋是指居民个人融人社区生活的程 度 ${ }^{[36]}$, 反映居民与社区在情感上的联系 ${ }^{[37-39]}$, 可通过 社区认同、社区依赖和社会关系来衡量 ${ }^{[40]}$ 。社区依 恋会影响居民感知。一般而言,社区依恋对成本感 知有负向影响, 对获益感知会产生正向的影响, 尤 其是在经济获益和社会获益方面 ${ }^{[41-43]}$ 。但在社区依 恋和旅游支持度关系方面,学者们的研究结论并不 一致。例如, Nicholas 等 ${ }^{[21]}$ 的研究发现,社区依恋直 接正向影响居民旅游支持度, 社区依恋程度越高, 越支持旅游发展。而 Choi 等 ${ }^{[4]}$ 的研究则得出相反 的结论。此外,还有学者认为社区依恋通过影响旅 游感知间接影响旅游支持度 ${ }^{[45]}$ 。基于以上的研究, 提出以下假设:

$\mathrm{H} 3$ : 社区依恋对旅游获益感知具有影响;

$\mathrm{H} 4$ : 社区依恋对旅游成本感知具有影响;

$\mathrm{H} 5$ :社区依恋对旅游支持度具有影响。

2.2.2 社区关注 (Community Concern, CC)

社区关注是指社区居民了解并关注社区的事 务, 是居民与社区关系的行动表现 ${ }^{[43]}$ 。社区关注是 居民感知与支持度的重要影响因素。研究表明,社 
区关注和获益感知之间存在积极的关系 ${ }^{[46]}$, 特别在 经济和文化获益感知方面, 社区关注具有显著的正 向影响 ${ }^{[42]}$ 。社区关注与居民之间的关系, 以往研究 主要以感知作为中介变量进行探讨 ${ }^{[47]}$, 如王纯阳 等 ${ }^{[22]}$ 针对福建土楼的案例研究发现, 社区关注通过 经济获益感知和环境获益感知间接正向影响态度， 并通过经济成本感知间接负向影响态度。但社区 关注与社区依恋作为居民一社区关系的平行测量 变量, 其对旅游支持度直接影响的研究尚未像社区 依恋一样引起足够的重视。

基于以上的研究和讨论,本文提出以下假设：

$\mathrm{H} 6$ : 社区关注对旅游获益感知具有影响;

$\mathrm{H} 7$ : 社区关注对旅游成本感知具有影响;

$\mathrm{H} 8$ : 社区关注对旅游支持度具有影响。

综上所述,本文提出究假设模型(图 1)。

\section{3 研究设计}

\section{1 问卷设计}

首先, 在回顾现有文献的基础上, 结合台湾地 区的特征和实际情况，初步设计调查问卷。其次， 邀请 5 位台湾东华大学观光游憩学专业研究生根据 台湾居民语言表达习惯对问卷中可能产生歧义, 表 达不清晰, 以及不符合台湾居民表达习惯的内容进 行修改。第三, 邀请6 位专家学者 (4位台湾学者和 2 位大陆学者)对问卷维度和题项设计的合理性提 出建议, 并完善问卷。第四, 于 2016年 3 月在花莲 市进行预调查, 以拦访方式调研了 60 余名居民, 并 根据预试问卷结果, 删除了分辨力不强的题项, 修 正了表达不清楚的用词。最终形成的问卷主体由 5 个维度 18 个题项构成, 分别为社区依恋、社区关注、 旅游获益感知、旅游成本感知和旅游支持度。

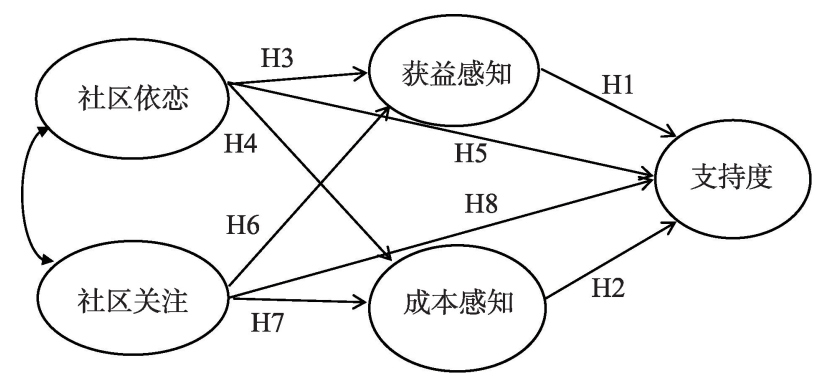

图 1 居民旅游支持度研究假设模型

Figure 1 A model of residents' support for tourism
社区依恋和社区关注量表参考了 Nicholas 等 ${ }^{[21]}$ 和王纯阳等 ${ }^{[2]}$ 的研究。社区依恋包括 “跟其他地区 相比,我更喜欢当地的自然环境” $(C A 1)$ 、“跟其他地 区相比,我更愿意居住在当地” $(C A 2)$ 、“当地对我成 长的意义重大” $(C A 3) 3$ 个题项。社区关注包括“我 关心当地自然环境的保护” $(C C 1)$ 、“我关心旅游发 展对当地的影响” ( $C C 2)$ 、“我关心当地的经济发展 动向” $(C C 3)$ 、“我关心当地公共设施的建设” $(C C 4)$ 4 个题项。

旅游获益感知和成本感知参考了 Gursoy 等 ${ }^{[46]}$ 和王纯阳等的研究 ${ }^{[22]}$ 。旅游获益感知包括“增加了 当地居民的就业机会” $(T B 1)$ 、“促进了当地经济的 发展”(TB2)、“提高了当地居民的经济收人” $(T B 3)$ 、 “提高了当地的知名度” $(T B 4) 4$ 个题项。旅游成本 感知包括“当地的优良传统受到不良冲击” $(T C 1)$ 、 “旅游发展直接或间接破坏了自然环境” $(T C 2)$ 、“旅 游业加速了对环境的污染” (TC3)、“旅游业造成本 地的交通拥挤问题”( TC4 4 4 个题项。

旅游支持度参考了 Oviedo- Garcia 等 ${ }^{[23]}$ 和 Nunkoo 等 ${ }^{[30]}$ 的研究,包括: “我支持大陆游客来台湾 旅游” (TS1)、“政府应鼓励大陆游客来台湾旅游” (TS2)、“大陆游客对台湾旅游业发展有积极影响” (TS3) 3 个题项。

以上所有维度的测量指标均采用李克特 5 级量 表评估, 1 5 分分别表示“完全不同意”、“不同意”、 “一般”、“同意”和“完全同意”。

受访者的基本信息部分包括性别、年龄、教育 程度、婚姻状况、职业、在当地居住的时间、收人 等。为了核实调研对象是否能区分和剥离大陆游 客这一群体的旅游影响感知和态度,还设计“您是 否有与大陆游客接触的经验” “您平均每月与大陆 游客互动频率” “您是否能区别大陆游客” 等 3 个题 项以提高问卷效度。共计 11 个题项。

\section{2 数据收集与样本设计}

本文以台湾地区作为调研区域,问卷发放地包 括台北市、高雄市 2 个行政“直辖市”,新北县、台北 县、台中县、高雄县等 15 个县/市。首先根据台湾统 计资讯网 ${ }^{[48}$ 统计的各县、市人口数量先进行分层抽 样,计算不同县、市应该发放问卷的数量; 其次根据 台湾观光部门 ${ }^{[49]}$ 及地方官方网站公布的必游景点排 
名, 选取各县、市热门景点、游客必经车站等附近的 社区作为发放问卷的调研地点 (分布详见表 1); 最 后根据便利抽样原则, 选择调研对象, 尽力涵盖不 同人口统计学特征的居民样本。

本文总计调研 25 个地点, 涉及社区居民总人口 约 7000 人, 按照 $5 \%$ 的抽样比例大约需要获取 350 份有效问卷。从2016年4-6月,由 2 名研究人员对 社区居民进行面对面问卷发放。总计发放问卷 400 份, 剥离出选项问题超过一半未回答或者连续选择 相同的选项, 以及自相矛盾的无效问卷, 对于漏项 较少的数据, 采用均值替换法进行插补。最终, 获 取有效问卷 341 份, 问卷有效率为 $85 \%$ 。

\section{4 结果与分析}

\section{1 样本人口统计学特征}

根据样本统计资料, 受访对象人口统计学特征 分布如表 2 所示。在性别结构上, 男性多于女性; 样 本群体以中青年为主; 服务业从业人员最多, 其次 是工商业人员; 有一半以上的受访者为专科及以上 学历; 月净收人 (新台币) 集中在 2 5万元; 有高达
$80.9 \%$ 的受访者与大陆游客有接触经验,一般每月 与大陆游客互动频率在 1 5 次之间。除了现场直接

表 1 调研问卷发放地点

Table 1 Locations of distributed survey questionnaire

\begin{tabular}{cl}
\hline 县/市 & \multicolumn{1}{c}{ 问卷发放地点 } \\
\hline 台北市 & 师大夜市、信义商圈、台北火车站附近 \\
高雄市 & 高雄火车站附近、高雄驳二艺术特区 \\
台北县 & 九份、野柳地质公园 \\
新北县 & 板桥夜市 \\
桃园县 & 桃园火车站附近 \\
台中县 & 台中火车站附近、忠孝夜市 \\
台南市 & 安平古堡、安平树屋 \\
苗栗县 & 苗栗火车站附近 \\
彰化县 & 彰化火车站附近 \\
南投县 & 日月潭景区 \\
云林县 & 云林火车站附近 \\
嘉义县 & 阿里山景区 \\
屏东县 & 量丁夜市 \\
花莲县 & 七星潭、太鲁阁 \\
新竹县 & 内弯老街 \\
宜兰县 & 几米广场 \\
台东县 & 伯朗大道、三仙台 \\
\hline &
\end{tabular}

表 2 样本人口统计学特征 $(N=341)$

Table 2 Respondents' profiles $(N=341)$

\begin{tabular}{|c|c|c|c|c|c|}
\hline 项目 & 类别 & 有效百分比 $/ / \%$ & 项目 & 类别 & 有效百分比 $/ \%$ \\
\hline \multirow[t]{2}{*}{ 性别 } & 男 & 53.6 & 学历 & 初中及以下 & 2.1 \\
\hline & 女 & 46.4 & & 高中、高职 & 26.8 \\
\hline \multirow[t]{7}{*}{ 年龄/岁 } & $\leqslant 18$ & 0.9 & & 大学、专科 & 53.9 \\
\hline & $19 \sim 23$ & 16.5 & & 研究生及以上 & 17.3 \\
\hline & $24 \sim 29$ & 20.9 & 月净收人/万新台币 & 无收人 & 8.1 \\
\hline & $30 \sim 39$ & 28.2 & & $\leqslant 2$ & 16.1 \\
\hline & $40 \sim 55$ & 27.9 & & $2 \sim 3$ (含) & 21.8 \\
\hline & $56 \sim 65$ & 4.1 & & 3 5(含) & 29.3 \\
\hline & $\geqslant 66$ & 1.5 & & 5 7(含) & 12.2 \\
\hline \multirow[t]{2}{*}{ 婚姻状况 } & 已婚 & 44.5 & & 7 10(含) & 7.5 \\
\hline & 未婚 & 55.5 & & $>10$ & 5.1 \\
\hline \multirow[t]{8}{*}{ 职业 } & 军人、公务员、教师 & 11.6 & 是否接触过大陆游客 & 是 & 80.9 \\
\hline & 农林渔牧 & 0.9 & & 否 & 19.1 \\
\hline & 工商业 & 19.3 & 每月与大陆游客 & $0 \sim 1$ & 29.2 \\
\hline & 家庭主妇 & 5.6 & 互动频率/次 & $2 \sim 5$ & 39.8 \\
\hline & 服务业 & 44.8 & & $6 \sim 10$ & 8.6 \\
\hline & 退休人员 & 1.2 & & $11 \sim 20$ & 5.3 \\
\hline & 学生 & 10.1 & & $21 \sim 30$ & 6.2 \\
\hline & 其他 & 6.5 & & $\geqslant 31$ & 10.9 \\
\hline
\end{tabular}

注: “每月与大陆游客互动频率” 按年接触次数除以 12 个月计算。 
接触, 受访者还通过新闻媒体等间接渠道对大陆游 客有所了解。即使是受访者与大陆游客直接接触 不多, 因为其所在社区距离景点较近, 对旅游发展 带来的变化还是有所体会。总体而言, 受访者对大 陆游客都有一定的直接或间接了解。

\section{2 信度和效度检验}

虽然测量的问题都来自于经典问卷, 并经过专 家审议,但是为了保证各个变量在同一维度下具有 一致性,研究者用Cronbach's $\alpha$ 系数进行了信度分 析, 同时还用因子分析的方法对各个题目进行了结 构效度的分析。信度结果显示, 总体的 Cronbach's $\alpha$ 系数为 0.821 , 各个维度的修正后总相关系数 $(C I T C)$ 都大于 0.5 , 量表的内部一致性比较好 (表 3)。因子分析的结果显示, 测量模型中每个观察变 量的标准化因子载荷大于 0.5 , 而且具有统计学上的 显著意义, 累计方差贡献率是 $71.1 \%, K M O$ 值为 0.826 , Bartlett球形检验的结果显著 $(p<0.001)$, 问卷 的结构效度较好 (表 4)。

为了检验模型的拟合情况, 确认数据模型是否 是研究预期形式, 首先进行测量模型的分析, 利用 SPSS 23.0 和 AMOS 20 软件对模型进行了验证性因

\section{表 3 调查问卷量表的信度检验}

Table 3 Reliability of the scale of questionnaire survey results

\begin{tabular}{ccc}
\hline 潜变量 & 校正的项总相关系数 & Cronbach’s $\alpha$ \\
\hline 社区依恋 & 0.574 & 0.801 \\
& 0.591 & \\
社区关注 & 0.592 & 0.835 \\
& 0.562 & \\
& 0.697 & \\
& 0.725 & \\
获益感知 & 0.681 & \\
& 0.681 & \\
& 0.761 & \\
& 0.734 & \\
成本感知 & 0.590 & 0.842 \\
& 0.542 & \\
& 0.766 & \\
& 0.769 & \\
支持度 & 0.644 & \\
& 0.801 & \\
& 0.826 & \\
& 0.683 & \\
\hline
\end{tabular}

子分析 (Confirmatory Factor Analysis, CFA)。在验 证性因子分析和结构方程模型分析中, 卡方检验 $\left(\chi^{2}\right.$ Test) 是最常用的模型评鉴方法, 在卡方检验中

表 4 调查问卷量表的因子分析结果

Table 4 Factor analysis of questionnaire survey results

\begin{tabular}{|c|c|c|c|c|}
\hline 潜变量 & 变量名 & 测量题项 & 标准化因子载荷 & 累计方差贡献率 $/ \%$ \\
\hline \multirow[t]{4}{*}{ 获益感知 } & $T B 1$ & 增加了当地居民的就业机会 & 0.759 & 27.206 \\
\hline & $T B 2$ & 促进了当地经济的发展 & 0.837 & \\
\hline & $T B 3$ & 提高了当地居民的经济收人 & 0.834 & \\
\hline & $T B 4$ & 提高了当地的知名度 & 0.645 & \\
\hline \multirow[t]{4}{*}{ 成本感知 } & $T C 1$ & 当地的优良传统受到不良冲击 & 0.600 & 46.532 \\
\hline & $T C 2$ & 旅游业直接或间接破坏了自然环境 & 0.851 & \\
\hline & $T C 3$ & 旅游业加速了旅游业对环境的污染 & 0.873 & \\
\hline & $T C 4$ & 旅游业造成本地的交通拥挤问题 & 0.645 & \\
\hline \multirow[t]{4}{*}{ 社区关注 } & $C C 1$ & 我关心当地自然环境的保护 & 0.586 & 60.003 \\
\hline & $C C 2$ & 我关心旅游发展对当地的影响 & 0.713 & \\
\hline & $C C 3$ & 我关心当地的经济发展动向 & 0.866 & \\
\hline & $C C 4$ & 我关心当地公共设施的建设 & 0.817 & \\
\hline \multirow[t]{3}{*}{ 社区依恋 } & $C A 1$ & 跟其他地区相比, 我更喜欢当地的自然环境 & 0.544 & 66.590 \\
\hline & $C A 2$ & 跟其他地区相比, 我更愿意居住在当地 & 0.884 & \\
\hline & $C A 3$ & 当地对我成长的意义重大 & 0.563 & \\
\hline \multirow[t]{3}{*}{ 支持度 } & $T S 1$ & 我支持大陆游客来台湾旅游 & 0.889 & 71.101 \\
\hline & $T S 2$ & 政府应鼓励大陆游客来台湾旅游 & 0.926 & \\
\hline & $T S 3$ & 大陆游客对台湾旅游业发展有积极影响 & 0.724 & \\
\hline
\end{tabular}


$p>0.05, \chi^{2} / d_{.} . f<3$ 说明模型具有解释度。除了卡方检 验, 为了检验模型的拟合度, 以及估计的假设模型 与理论抽样导出的卡方值之间的差异程度, 还应使 用模型拟合指数和替代指数进行检验。最常见的 模型拟合指数主要包括拟合指数 (Goodness-of-fit Index, GFI)、调节括拟合指数 (Adjusted GFI, AGFI)、正规拟合指数 (Normed Fit Index, NFI)、非规准 适配指数 (Tacker-Lewis Index, TLI)。模型拟合指数 介于 0 和 1 之间, 指数越接近 1 , 说明模型拟合度越 高, GFI、AGFI、NFI、TLI 的门槛值一般为 $0.8^{[50,51]}$ 。替 代指数主要包括平均概似平方误根系数 (Root Mean Error of Approximation, RMSEA）、非集中性 参数 (Non-Centrality Parameter, NCP)、Akaike 讯息 指数 (Akaike Information Criterion, AIC), 数值越小 越好, 其中 RMSEA 不受样本大小和模型复杂度的 影响, 是重要的替代指数指标 ${ }^{[52]}$ 。RMSEA 介于 0 和 1 之间,数值越小越好, 可接受的值为 0.05 到 0.08 ; $\mathrm{NCP} 、 \mathrm{AIC}$ 的数值没有范围, 但是越小越好 ${ }^{[53]}$ 。

根据上述指标体系, 本研究的验证性因子分析 数据拟合度显示所有的拟合度指标都高于推荐指 标 $\left(\chi^{2}=323.065\right.$, d.f. $=125, \chi^{2} /$ d.f. $=2.585, G F I=0.901$, $A G F I=0.865, \quad R M S E A=0.068, \quad N F I=0.900, \quad T L I=$ 0.921 ), 即卡方值与自由度之比小于 $3, G F I 、 A G F I$ 、 $N F I 、 T L I$ 值都大于 $0.8, R M S E A$ 值小于 0.08 , 说明数 据与测量模型之间有较好的拟合度。组合信度 $C R$ 值大于 0.7 (表 5$)$,符合 Hair 等 ${ }^{[54]}$ 学者提出的指标建 议,模型的收玫效度较好。

表 6 显示了各个变量的相关系数矩阵,括号中 的数据是 $R^{2}$ 。对比发现, 每个潜变量的平均提炼方 差值 $(A V E)$ 都大于对应的 $R^{2}$, 即各个变量之间具有 区别效度 ${ }^{[5]}$ 。量表的信度和效度都通过了检验。
表 5 调查问卷中各观察变量的因子载荷

Table 5 Factor loading of each observed variable

\begin{tabular}{cccc}
\hline 潜变量 & 变量名 & $t$ 值 & 组合信度 \\
\hline 社区依恋 & $C A 1$ & 7.815 & 0.886 \\
& $C A 2$ & 8.464 & \\
社区关注 & $C A 3$ & - & \\
& $C C 1$ & 10.325 & 0.835 \\
& $C C 2$ & 13.140 & \\
获益感知 & $C C 3$ & 17.702 & \\
& $C C 4$ & - & \\
& $T B 1$ & - & 0.854 \\
& $T B 2$ & 15.330 & \\
成本感知 & $T B 3$ & 15.305 & \\
& $T B 4$ & 11.566 & \\
& $T C 1$ & 10.142 & 0.837 \\
& $T C 2$ & 14.094 & \\
& $T C 3$ & 15.409 & \\
& $T C 4$ & - & 0.712 \\
支持度 & $T S 1$ & - & \\
& $T S 2$ & 23.927 & \\
& $T S 3$ & 16.005 & \\
\hline
\end{tabular}

\section{3 结构方程模型分析}

用最大似然法对模型进行结构方程分析, 模型 拟合度如下: $\chi^{2}=328.639$, d.f. $=126, \chi^{2} / d . f .=2.608$, $G F I=0.901, A G F I=0.865, R M S E A=0.069, N F I=0.898$, $T L I=0.920,8$ 个假设中 5 个成立, 3 个不成立(图 2)。

检验结果支持的因果关系假设包括(表 7): 社 区依恋对获益感知产生负向影响;社区关注对获益 感知产生正向影响; 社区关注对支持度产生正向影 响; 获益感知对支持度产生正向影响; 成本感知对 支持度产生正向影响。

检验结果不支持的因果关系包括:社区依恋对 支持度的正向影响,社区依恋对成本感知的负向影 响,社区关注对成本感知的负向影响。根据AMOS

表 6 各研究变量之间的相关系数矩阵及 $A V E$ 值

Table 6 Correlations among study constructs and average variance extracted (AVE)

\begin{tabular}{|c|c|c|c|c|c|}
\hline 潜变量 & 支持度 $(T S)$ & 成本感知 $(T C)$ & 获益感知 $(T B)$ & 社区关注 $(C C)$ & 社区依恋 $(C A)$ \\
\hline 支持度 $(T S)$ & 1.000 & & & & \\
\hline 成本感知 $(T C)$ & 0.418 & 1.000 & & & \\
\hline 获益感知 $(T B)$ & 0.730 & 0.153 & 1.000 & & \\
\hline 社区关注 $(C C)$ & 0.237 & -0.007 & 0.118 & 1.000 & \\
\hline 社区依恋 (CA) & 0.058 & -0.056 & -0.042 & 0.609 & 1.000 \\
\hline 平均提炼方差 $(A V E)$ & 0.724 & 0.566 & 0.597 & 0.567 & 0.465 \\
\hline
\end{tabular}




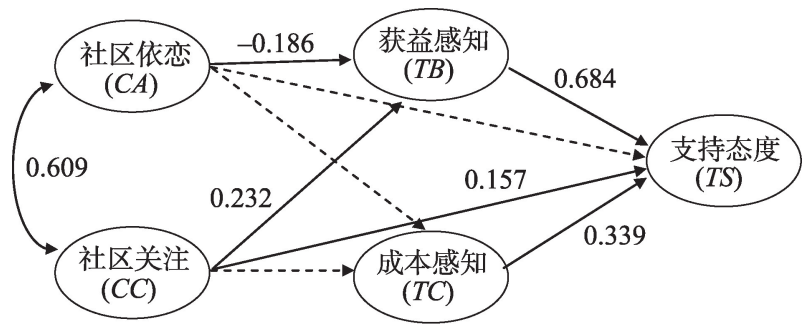

图 2 结构方程模型分析结果 $(N=\mathbf{3 4 1})$

Figure 2 Results of structural equation model $(N=341)$ 注: 实线箭头表示通过假设检验; 虚线箭头表示未通过假设检验。

表 7 研究假设检验结果 $(N=341)$

Table 7 Result of maximum-likelihood parameter estimates $(N=341)$

\begin{tabular}{lccc}
\hline \multicolumn{1}{c}{ 假设路径 } & 标准化回归系数 & $t$ 值 & 假设 \\
\hline $\mathrm{H} 1:$ 获益感知 $\rightarrow$ 支持度 & 0.684 & $11.827^{* *}$ & 成立 \\
$\mathrm{H} 2:$ 成本感知 $\rightarrow$ 支持度 & 0.339 & $7.123^{* *}$ & 成立 \\
$\mathrm{H} 3$ : 社区依恋 $\rightarrow$ 获益感知 & -0.186 & $-2.066^{*}$ & 成立 \\
$\mathrm{H} 4:$ 社区依恋 $\rightarrow$ 成本感知 & -0.096 & -1.080 & 不成立 \\
$\mathrm{H} 5:$ 社区依恋 $\rightarrow$ 支持度 & 0.012 & 0.187 & 不成立 \\
$\mathrm{H} 6:$ 社区关注 $\rightarrow$ 获益感知 & 0.232 & $2.664^{*}$ & 成立 \\
$\mathrm{H} 7:$ 社区关注 $\rightarrow$ 成本感知 & 0.056 & 0.652 & 不成立 \\
$\mathrm{H} 8:$ 社区关注 $\rightarrow$ 支持度 & 0.157 & $2.544^{*}$ & 成立 \\
\hline
\end{tabular}

注: **表示 $p<0.01$, *表示 $p<0.05$ 。

输出结果, 获益感知内生变量被社区依恋和社区关 注解释度为 $3.6 \%\left(R^{2}=0.036\right)$, 支持度内生变量被获 益感知、社区关注和成本感知解释度为 $63.8 \%\left(R^{2}=\right.$ $0.638)$ 。

研究者在研究之前先进行了预调查, 预调查过 程中对问卷进行了反复修改, 并剔除了分辨度不高 的问题。在正式调查后也对无效问卷进行了处理, 数据质量比较理想, 结构方程的拟合度指数符合模 型分析要求,故没有再进行进一步的修正处理。

\section{4 效应分析}

从图 2 可以看出, 在经过检验成立的假设路径
中, 支持度除受到获益感知的直接影响, 社区依恋 还通过获益感知对支持度产生了间接影响。此外， 社区关注不仅直接对支持度有影响, 还通过获益感 知对支持度产生了间接影响。分别分析几条路径 的直接效应和间接效应(表 8),并对各条路径效应 的显著性分别用 Sobel、Aroian 和 Goodman 等 3 种方 法进行了检验(表9)。社区依恋通过获益感知对支 持度的间接效应为 -0.148 , 有负向的影响, 即居民的 社区依恋会通过他们的获益感知来影响旅游支持 度。社区依恋度越高, 获益感知越低, 旅游支持度 也越低, 3 种检验 $p$ 值都小于 0.05 , 间接效应显著。 社区关注对支持度产生的直接效应是 0.211 ,社区关 注通过获益感知对支持度产生的间接效应是 0.124 , 总计效应为 $0.335,3$ 种检验的 $p$ 值都小于 0.01 , 间接 效应显著。即居民的社区关注也会通过他们的获 益感知来影响旅游支持度, 居民的社区关注越高， 获益感知度就越高, 旅游支持度也越高。此外社区 关注的提高, 也会直接提升居民旅游支持度。

\section{5 结论、讨论与展望}

\section{1 结论与讨论}

(1) 台湾居民的旅游获益感知对大陆赴台旅游 支持度有正向影响,研究假设 $\mathrm{H} 1$ 成立。台湾居民 认为大陆游客赴台旅游最主要的积极影响是促进 当地经济发展、经济收人提高以及提供工作机会。 这一结论与台湾、大陆学者的研究基本一致,也与 台湾观光部门的调查情况相符。台湾学者的研究

\begin{tabular}{|c|c|c|c|}
\hline 路径 & 直接效应 & 间接效应 & 总效应 \\
\hline 社区依恋 一支持度 & & -0.148 & -0.148 \\
\hline 社区关注一支持度 & 0.211 & 0.124 & 0.335 \\
\hline
\end{tabular}

表 9 获益感知的中介效应显著性检

Table 9 Mediation tests of tourism benefit

\begin{tabular}{|c|c|c|c|c|c|c|}
\hline 路径 & 变量关系 & 非标准化回归系数 & 标准差 & Sobel检验 & Aroian检验 & Goodman 检验 \\
\hline \multirow[t]{2}{*}{ 社区依恋 $\rightarrow$ 获益感知一支持度 } & $C A \rightarrow T B$ & -0.259 & 0.125 & $-2.041 *$ & $-2.033^{*}$ & $-2.048 *$ \\
\hline & $T B \rightarrow T S$ & 0.764 & 0.065 & & & \\
\hline \multirow[t]{2}{*}{ 社区关注 $\longrightarrow$ 获益感知 $\longrightarrow$ 支持度 } & $C C \rightarrow T B$ & 0.280 & 0.105 & $2.600 * *$ & $2.592 * *$ & $2.610 * *$ \\
\hline & $T B \rightarrow T S$ & 0.764 & 0.065 & & & \\
\hline
\end{tabular}

注: "*表示 $p<0.01, *$ 表示 $p<0.05$ 。 
结果充分肯定了大陆赴台旅游对于台湾经济、就业 与地方繁荣发展的推动作用 ${ }^{[5,57]}$ 。这一研究假设部 分印证了大陆学者的研究结论。如黄东政 ${ }^{[18]}$ 基于台 湾居民正面影响集群的研究,对比了乐观期待集 群、认同期许集群、经济优先集群对台湾发展大陆 居民赴台旅游持支持度。台湾观光部门对大陆游 客的调查显示, 大陆旅客对 “台湾民众态度友善”满 意度的平均值较高, 达到 4.69 (满分为 5 分), 可间接 地反映出台湾居民因获益感知而表现出对大陆游 客友善和积极的态度 ${ }^{[58]}$ 。

(2)台湾居民的旅游成本感知对大陆赴台旅游 支持度产生影响, 且影响为正向 (即研究假设 H2)。 问卷调查显示: “旅游发展直接或间接破坏了自然 环境” “旅游业提高了本地物价”为台湾居民对旅游 成本最为显著的感知, 说明台湾居民最为关注环境 与经济方面的影响, 对社会文化方面的影响感知不 明显。台湾居民担忧的主要内容为大陆游客的增 长对环境承载力的挑战以及不文明行为对于环境 的破坏 ${ }^{[17,18]}$, 但目前并未有相关研究与报道表明环 境超载与不可逆资源破坏现象的发生。同时,对高 雄房价的实证研究表明, 大陆游客赴台对包括房价 在内的台湾物价并未产生影响 ${ }^{[99]}$ 。在调查中发现， 对旅游的成本感知比较明显的居民, 在旅游业中的 参与度一般也较高。切实的经济收益使得居民对 旅游发展持更加积极的态度。相关研究也表明, 即 便居民可能对当前的发展有不满意的地方, 如果居 民仍对旅游的未来发展充满期盼, 也会支持本地旅 游的发展 ${ }^{[29,60]}$ 。

(3) 台湾居民的社区关注对旅游支持度存在直 接影响, 且影响为正向 (即研究假设 H8)。这 2 个变 量之间的关系在以往的研究中涉及不多。如前文 所述, 以往的研究结论多为社区关注通过旅游感知 间接影响旅游支持度 ${ }^{[22,47]}$ 。但是本文发现社区关注 对旅游支持度具有直接影响。社区关注体现了居 民对社区的了解和关心。一方面, 发展旅游作为目 的地社区重要的事务, 获得了对社区关注程度高的 居民支持; 另一方面, 在访谈中, 台湾居民认为虽然 随着时代发展邻里关系发生了变化, 但并没有将之 “归各”于旅游业。这一点与梁冰瑜等 ${ }^{[61]}$ 在丹霞山的 研究结果一致。与此同时, 社区关注对旅游获益感
知有正向影响 (即研究假设 H6)。即居民对社区的 关注度越高, 越能感受到旅游发展的正面影响,进 而支持旅游发展,印证了王纯阳等关于社区关注通 过经济与环境获益感知间接正向影响态度的结 论 ${ }^{[22]}$ 。由此可见,社区关注对于提升居民的旅游发 展支持度具有非常积极的影响作用。但社区关注 对于旅游成本感知没有显著影响 (即研究假设 $\mathrm{H} 7$ 不成立)。

(4) 台湾居民的社区依恋对旅游获益感知有负 向影响, 研究假设 H3 成立。居民对社区的依恋程 度越高, 越容易聚焦旅游发展带来的负面影响, 因 此对旅游获益感知较弱, 间接负向影响支持度。这 一结论分别印证了 Um 等 ${ }^{[2]}$ 和 Gursoy 等 ${ }^{[41]}$ 的研究结 果, 即具有强烈依恋感的社区居民更趋向于负面评 价旅游发展带来的影响, 同时社区依恋通过影响旅 游感知间接影响旅游支持度。但本文的结论与王 纯阳等 ${ }^{[22]}$ 的研究发现相左,他的研究认为社区依恋 对旅游获益影响感知有积极影响。另一方面, 社区 依恋对旅游成本感知与旅游支持度均未产生直接 影响 (即研究假设 H4 和 H5 不成立)。这与 Gursoy 等认为的社区依恋对成本感知有负向影响 ${ }^{[4]}$ 、Nicholas 等认为的社区依恋对旅游支持度有直接正向影 响的结论不同 ${ }^{[21]}$ 。

\section{2 展望}

(1)海峡两岸基于旅游发展所进行的合作与互 动, 在两岸关系和平发展进程中具有机制构建、利 益联结、文化整合及社会融合等政治功能 ${ }^{[3]}$, 能在深 化两岸利益融合、共创两岸互利双赢、增进两岸同 胞福祉、推动两岸关系和平发展方面发挥重要作 用。尤为重要的是, 调研中发现, 大陆赴台旅游对 于台湾居民对大陆现代化发展以及青年一代的理 解与认识能发挥积极作用。台湾居民的支持, 是推 动大陆赴台旅游有序发展的重要力量。而本文通 过对社区依恋、社区关注、影响感知与支持度的结 构模型构建, 获得了台湾居民的支持度受到多种因 素影响的结论,特别是台湾居民的社区依恋和社区 关注都会通过影响获益感知这个中介变量来间接 影响他们对大陆游客赴台旅游的支持度等结论。 上述结论有助于为相关旅游政策制定找到合适的 突破口。因此,需要利用适当的方式,通过具体数 
字和生动案例, 向台湾民众展现大陆赴台旅游对于 解决台湾基层民众与青年就业, 扩大台湾旅行社、 民宿等产业发展空间,保护、活化与传播当地文化， 激发社区活力,优化基础设施等方面的积极作用, 努力扩大台湾民众参与越多受益越多的获得感, 进 一步争取台湾同胞对于两岸经济社会融合发展的 理解与支持。2018 年台湾县市长选举结果是态度 影响行为,进而可能左右政策的具体体现, 反映了 台湾同胞对于持续与深化两岸合作的期盼, 将助益 两岸旅游合作机制的优化、范围的扩展、层级的提 升,从而提升台湾民众的受益面和受益度。

(2)本文将大陆游客作为独立的研究对象, 探 究台湾居民针对大陆游客赴台旅游影响和态度的 感知,丰富了以往不区分游客类型的相关研究。未 来的研究既可以从不同游客群体比较,也可以从社 区居民人口统计学特征分类, 以及结合身份认同等 影响因素等视角,细化台湾地区社区居民支持度的 分析,从而为决策制定提供更为有益的理论依据。

\section{参考文献(References):}

[1] Sharpley R. Host perceptions of tourism: A review of the research [J]. Tourism Management, 2014, 42: 37-49.

[2] 汪侠, 甄峰, 吴小根, 等. 旅游开发的居民满意度驱动因素: 以广 西阳朔县为例 [J]. 地理研究, 2010, 29(5): 841-851. [Wang X,

Zhen F, Wu X G, et al. Driving factors of resident satisfaction with tourism development: A case study of Yangshuo in Guangxi Zhuang Autonomous Region[J]. Geographical Research, 2010, 29 (5): 841-851.]

[3] 尹立杰, 张捷, 韩国圣, 等. 基于地方感视角的乡村居民旅游影 响感知研究: 以安徽省天堂寨为例[J]. 地理研究, 2012, 31(10): 1916-1926. [Yin L J, Zhang J, Han G S, et al. The research of rural residents' perception on tourism development impact from the sense of place perspective: A case study of Tiantangzhai in Anhui Province[J]. Geographical Research, 2012, 31(10): 1916-1926.]

[4] 卢春天, 石金莲. 旅游地居民感知和态度研究现状与展望[J]. 旅 游学刊, 2012, 27(11): 32-43. [Lu C T, Shi J L. Study on residents' perceptions and attitude in tourist destinations: Current state and prospect[J]. Tourism Tribune, 2012, 27(11): 32-43.]

[5] 吴丽敏, 黄震方, 谈志娟, 等. 江南文化古镇居民旅游影响感知 及其形成机理: 以同里为例[J. 人文地理, 2015, 30(4): 143148. [Wu L M, Huang Z F, Tan Z J, et al. Tourism impact perception of residents in Jiangnan ancient town and its formation mecha- nism: A case study of Tongli[J]. Human Geography, 2015, 30(4): $143-148$.

[6] 汲忠娟, 蒋依依, 谢婷. 旅游地居民感知和态度研究综述 [J]. 资 源科学, 2017, 39(3): 396-407. [Ji Z J, Jiang Y Y, Xie T. The perceptions and attitudes of residents towards the impacts of tourism [J]. Resources Science, 2017, 39(3): 396-407.]

[7] Ap J, Crompton J L. Developing and testing a tourism impact scale [J]. Journal of Travel Research, 1998, 37(2): 120-130.

[8] 汪德根, 王金莲, 陈田, 等. 乡村居民旅游支持度影响模型及机 理: 基于不同生命周期阶段的苏州乡村旅游地比较 $[\mathrm{J}]$. 地理学 报, 2011, 66(10): 1413-1426. [Wang D G, Wang J L, Chen T, et al. Influence model and mechanism of the rural residents for tourism support: A comparison of rural destinations of Suzhou in different life cycle stages[J]. Acta Geographica Sinica, 2011, 66(10): 1413-1426.]

[9] Sun Y Y, Pratt S, Yeoman I S, et al. The economic, carbon emission, and water impacts of Mainland visitors to Taiwan: Eco-efficiency and impact evaluation[J]. Journal of Travel Research, 2014, 53(6): 733-746

[10] Látková P, Vogt C A. Residents`́ attitudes toward existing and future tourism development in rural communities[J]. Journal of Travel Research, 2012, 51(1): 50-67.

[11] 王咏, 陆林. 基于社会交换理论的社区旅游支持度模型及应用: 以黄山风景区门户社区为例[J]. 地理学报, 2014, 69(10): 15571574. [Wang Y, Lu L. Community tourism support model and its application based on social exchange theory: Case studies of gateway communities of Huangshan Scenic Area[J]. Acta Geographica Sinica, 2014, 69(10): 1557-1574.]

[12] Husbands W. Social status and perception of tourism in Zambia[J]. Annals of Tourism Research, 1989, 16(2): 237-253.

[13] Lindberg K, Johnson R L. Modelling resident attitudes toward tourism[J]. Annals of Tourism Research, 1997, 24(2): 402-424.

[14] 台湾“交通部观光局”. 近十年(98 107)日、韩、马、大陆、港来台 旅客总人次变化[EB/OL]. (2019-02-23)[2019-04-23]. https:// stat.taiwan.net.tw/. [Tourism Bureau of Taiwan. The Total Number of Tourists Change from Japan, Korea, Malaysia, mainland China and Hong Kong in the Past Decade(98 107)[EB/OL]. (2019-0223)[2019-04-23]. https://stat.taiwan.net.tw/.]

[15] 林子荣, 李文献. 统一与对立: 两岸关系矛盾体演进研究(19492017)[J]. 台湾研究, 2018, (4): 26-36. [Lin Z R, Li W X. Analysis of the evolution of cross-strait relations since 1949 by using the method of contradiction analysis [J]. Taiwan Studies, 2018, (4): 26-36.]

[16] 林嘉慧, 彭素玲. 陆客来台观光驱动台湾旅游产业之转变[J]. 经 济前瞻, 2015, 1(157): 121-124. [Lin J H, Peng S L. Mainland tourists to visit Taiwan driving transformation of Taiwan's tourism industry[J]. Journal of Economic Perspectives, 2015, 1(157): 121- 
124.]

[17] 陈锦玉. 大陆来台旅游市场之旅游影响感知与态度初探 [J]. 岛 屿观光研究, 2013, 6(1): 49-71. [Chen J Y. A preliminary study on perceptions of tourism impacts and attitudes toward inbound travel market from Mainland China to Taiwan[J]. Island Tourism Research, 2013, 6(1): 49-71.]

[18] 黄东政, 黄福才. 大陆居民赴台旅游影响研究: 基于台湾居民感 知的视角 [J]. 旅游科学, 2009, 23(3): 48-54. [Huang D Z, Huang F C. An empirical study on the impacts of Mainland tourists to Taiwan: A Taiwan residents' perception view[J]. Tourism Science, 2009, 23(3): 48-54.]

[19] 陈鹏, 翟媛. 台湾居民对大陆游客旅游影响的感知与态度研究 [J]. 台湾研究, 2014, (4): 76-84. [Chen P, Zhai Y. Study on Taiwan residents' perception and attitude of the Mainland tourists impact[J]. Taiwan Studies, 2014, (4): 76-84.]

[20] 王新建, 郑向敏. 台湾居民对大陆游客感知的多维分析 [J]. 西南 民族大学学报(人文社科版), 2015, 36(1): 159-163. [Wang X J, Zheng X M. Multidimensional analysis of Taiwan residents' perception to mainland tourists[J]. Journal of Southwest Minzu University(Humanities and Social Science), 2015, 36(1): 159-163.]

[21] Nicholas L, Thapa B, Ko Y. Residents' perspectives of a world heritage site: The Pitons Management Area, St. Lucia[J]. Annals of Tourism Research, 2009, 36(3): 390-412.

[22] 王纯阳, 屈海林. 村落遗产地社区居民旅游发展态度的影响因 素 [J]. 地理学报, 2014, 69(2): 278-288. [Wang C Y, Qu H L. Study on influencing factors of residents' attitudes towards tourism development of village heritage sites[J]. Acta Geographical Sinica, 2014, 69(2): 278-288.]

[23] Oviedo-Garcia M A, Castellanos-Verdugo M, Martin-Ruiz D. Gaining residents' support for tourism and planning[J]. Internation -al Journal of Tourism Research, 2008, 10(2): 95-109.

[24] 程绍文, 张捷, 徐菲菲, 等. 自然旅游地社区居民旅游发展期望 与旅游影响感知对其旅游态度的影响: 对中国九寨沟和英国 NF 国家公园的比较研究[J]. 地理研究, 2010, 29(12): 21792188. [Cheng S W, Zhang J, Xu F F, et al. Effect of residents' tourism development expectation and tourism impacts perception on their attitude towards tourism in natural tourist destination: A comparative study between China's Jiuzhaigou and the UK's New Forest National Parks[J]. Geographical Research, 2010, 29(12): 2179-2188.]

[25] 彭建, 王剑. 旅游研究中的三种社会心理学视角之比较 [J]. 旅游 科学, 2012, 26(2): 1-9. [Peng J, Wang J. A comparative analysis of the three social psychological perspectives in tourism research [J]. Tourism Science, 2012, 26(2): 1-9.]

[26] Pavlos P, Konstantinos A. Altruism in tourism: Social exchange theory vs altruistic surplus phenomenon in host volunteering[J]. Annals of Tourism Research, 2017, 62: 26-37.
[27] Homans G C. Social behaviour as exchange[J]. American Journal of Sociology, 1958, 63(3): 597-606.

[28] Coulson A B, MacLaren A C, McKenzie S, et al. Hospitality codes and social exchange theory: The Pashtunwali and tourism in Afghanistan[J]. Tourism Management, 2014, 45: 134-141.

[29] Andereck K L, Valentine K M, Knopf R C, et al. Residents' perceptions of community tourism impacts[J]. Annals of Tourism Research, 2005, 32(4): 1056-1076.

[30] Nunkoo R, Ramkissoon H. Developing a community support model for tourism[J]. Annals of Tourism Research, 2011, 38(3): 964988.

[31] Lee T H. Influence analysis of community resident support for sustainable tourism development[J]. Tourism Management, 2013, 34 (1): $37-46$

[32] Ap J. Residents' perceptions on tourism impacts[J]. Annals of Tourism Research, 1992, 19(4): 665-690.

[33] 章锦河. 古村落旅游地居民旅游感知分析: 以黟县西递为例 [J]. 地理与地理信息科学, 2003, 19(2): 105-109. [Zhang J H. Research on ancient village residents' perceptions of tourism impact: A case of Village Yixian-Xidi[J]. Geography and Geo-Information Science, 2003, 19(2): 105-109.]

[34] Vargassánchez A, Plazamejía M D L Á, Porrasbueno N. Understanding residents' attitudes toward the development of industrial tourism in a former mining community[J]. Journal of Travel Research, 2009, 47(3): 373-387.

[35] Toennies F. Community and Society: Gemeinschaft and Gesellschaft[M]. East Lansing, MI: The Michigan State University Press, 1957.

[36] Trentelman C K. Place attachment and community attachment: A primer grounded in the lived experience of a community sociologist [J]. Society \& Natural Resources, 2009, 22(3): 191-210.

[37] Nunkoo R, Gursoy D. Residents' support for tourism an identity perspective[J]. Annals of Tourism Research, 2012, 39(1): 243268.

[38] Goudy W J. Community attachment in a rural region[J]. Rural Sociology, 1990, 55(2): 178-198.

[39] Theodori G L. Community attachment, satisfaction, and action[J]. Community Development, 2004, 35(2): 73-86.

[40] Harrill R, Potts T D. Tourism planning in historic districts: Attitudes toward tourism development in Charleston[J]. Journal of the American Planning Association, 2003, 69(3): 233-244.

[41] Gursoy D, Jurowski C, Uysal M. Resident attitudes: A structural modelling approach[J]. Annals of Tourism Research, 2002, 29(1): 79-105.

[42] Gursoy D, Chi C G, Dyer P. Locals' attitudes toward mass and alternative tourism: The case of sunshine coast, Australia[J]. Journal of Travel Research, 2010, 49(3): 381-394. 
[43] Jackson M S, Inbakaran R J. Evaluating residents' attitudes and intentions to act toward tourism development in regional Victoria, Australia[J]. International Journal of Tourism Research, 2006, 8 (5): 355-366.

[44] Choi H C, Murray I. Resident attitudes toward sustainable community tourism[J]. Journal of Sustainable Tourism, 2010, 18(4): 575594.

[45] Jackson M S, Inbakaran R J. Evaluating residents' attitudes and intentions to act toward tourism development in regional Victoria, Australia[J]. International Journal of Tourism Research, 2006, 8 (5): 355-366.

[46] Gursoy D, Kendall K W. Hosting mega events e modelling locals support[J]. Annals of Tourism Research, 2006, 33(3): 603-623.

[47] Perdue R R, Long P T, Allen L. Rural resident tourism perceptions and attitudes[J]. Annals of Tourism Research, 1987, 14(3): 420-429.

[48] 政府统计资讯网. 户籍人口统计速报[EB/OL]. (2016-03-06) [2018-07-22]. http://stat.ncl.edu.tw/hypage.cgi?HYPAGE=search/ detail.hpg\&dtd_id=1\&sysid=00008695. [Government Statistics. Statistic Report of Household Census[EB/OL]. (2016-03-06) [2018-07-22]. http://stat.ncl.edu.tw/hypage.cgi?HYPAGE=search/ detail.hpg\&dtd_id=1\&sysid=00008695.]

[49] 台湾 “交通部观光局”. 县市游咊据点[EB/OL]. (2016-03-26) [2016-04-06]. https://stat.taiwan.net.tw/scenicSpot. [Tourism Bureau of Taiwan. Scenic Spot of Each County[EB/OL]. (2016-0326)[2016-04-06]. https://stat.taiwan.net.tw/scenicSpot.]

[50] Bagozzi R P, Yi Y. On the evaluation of structural equation models [J]. Journal of the Academy of Marketing Science, 1988, 16(1): 74 -94 .

[51] Kline R B. Principles and Practice of Structural Equation Modelling. 2nd ed.[M]. New York: Guilford Press, 2005.

[52] Hu L T, Bentler P M. Cotoff criteria for fit indexes in covariance [J]. Structural Equation Modelling, 1991, 6(1): 1-55.

[53] Browne M W, Cudeck R. Alternative ways of assessing model fit [J]. Sociological Methods \& Research, 1992, 21(2): 230-258.

[54] Hair J F, Black W, Babin B, et al. Multivariate Data Analysis. 6th ed.[M]. New Jersey: Pearson Prentice Hall Press, 2005.

[55] Fornell C, Larcker D F. Evaluating structural equation models with unobservable variables and measurement error[J]. Journal of
Marketing Research, 1981, 18(3): 375-381.

[56] 张淑满. 开放陆客来台观光对台湾经济影响之一般均衡分析 [J]. 台北海洋技术学院学报, 2011, 4(2): 123-144. [Zhang S M.

The impacts of the opening for tourists from Mainland China on Taiwan's economy[J]. Journal of Taipei College of Marine Technology, 2011, 4(2): 123-144.]

[57] 黄园淋. 陆客来台观光及其政经影响(2008-2012)[D]. 衡阳: 南 华大学, 2013. [Huang Y L. The Economic and Political Influence of Mainland Tourists to Visit Taiwan(2008-2012)[D]. Hengyang: Nanhua University, 2013.]

[58] 中国新闻网. 台湾去年观光收人超百亿美元大陆客满意度最高 [EB/OL]. (2012- 08- 17) [2018- 08-22] .http://www.chinanews. com/tw/2012/08-17/4114493.shtml. [China News. The revenue of Taiwan tourism exceeded 10 billion dollars last year, Mainland tourists have the highest travel satisfaction[EB/OL]. (2012-08-17) [2018- 08- 22]. http://www.chinanews.com/tw/2012/08- 17/ 4114493.shtml.]

[59] 陈彦羽. 高雄捷运通车对区域房价之影响: 兼论陆客来台之影 响[D]. 桃园: “国立中央大学”, 2015. [Chen Y Y. The Effects of the Kaohsiung MRT Opened to the Regional House Prices: And the Effect of Mainland Tourists to Taiwan[D]. Taoyuan: "National Central University”, 2015.]

[60] Andriotis K, Vaughan D R. Urban residents' attitudes towards tourism development: The case of Crete[J]. Journal of Travel Research, 2003, 42(2): 172-185.

[61] 梁冰瑜, 彭华, 翁时秀. 旅游发展对乡村社区人际关系的影响 研究: 以丹霞山为例 [J]. 人文地理, 2015, 30(1): 129-134. [Liang B Y, Peng H, Weng S X. Study of the impact of tourism development to the interpersonal relationship in rural society: Cases from Danxiashan scenic spot[J]. Human Geography, 2015, 30(1): 129-134.]

[62] Um S, Crompton J L. Measuring resident's attachment levels in a host community[J]. Journal of Travel Research, 1987, 26(1): 2729.

[63] 吴乐杨. 旅游与政治: 观光旅游在两岸关系和平发展中的角色 功能 [J]. 湖北行政学院学报, 2013, (5): 36-40. [Wu L Y. Tourism and politics: The role and function of sightseeing tours in the peaceful development of cross-strait relations[J]. Journal of Hubei Administration Institute, 2013, (5): 36-40.] 


\title{
Attitude of Taiwan residents towards tourism from China's mainland
}

\author{
JIANG Yiyi' ${ }^{1,2}$, JI Zhongjuan ${ }^{3}$, XIE Ting ${ }^{4}$ \\ (1. School of Sports Recreation and Tourism, Beijing Sport University, Beijing 100084, China; 2. China Tourism Academy, \\ Beijing 100005, China; 3. School of Tourism Management, Sun Yat-sen University, Zhuhai 519082, China; 4. Department of \\ Tourism and MICE, Beijing Institute of Petro-chemical Technology, Beijing 102617, China)
}

\begin{abstract}
Since the official opening of mainland residents' travel to Taiwan in 2008, the mainland of China has gradually become an important tourist source market of Taiwan. The support of residents in Taiwan will not only affect the perception and satisfaction of mainland tourists, but also largely influence the tourism market policy between mainland and Taiwan, thus affect the normal communication between the two sides. It is of great academic value and important practical significance to understand Taiwan residents' support of mainland tourists to Taiwan. To reveal the impact of destination residents' attitude to tourism development, this research built a structural equation model between community attachment, community concern, residents' perceived benefit, residents' perceived cost, and support of tourism based on social exchange theory. An on- site questionnaire survey was used to collect data. After excluding incomplete and unusable responses, a total of 341 usable questionnaires were retained for the analysis in SPSS23.0 and AMOS 20. The results of the study indicate that residents' perceived benefit, perceived cost of tourism, and community concern were positively associated with their support of tourism. Community concern had both direct and indirect effects on attitude and it also affected tourism support attitude through perceived benefit. Community attachment had negative effect on tourism support attitude through perceived benefit. The interaction between community attachment and community concern did not positively affect their support. The relationship between Taiwan residents and community had both direct and indirect impacts on tourism development support. Tourism related policy attaches great importance to the protection of residents and community relations, and actively improve the residents' participation in tourism development. Tourism managers can improve residents' support for tourism development by improving the relationship between residents and communities. With increased awareness of the positive role of mainland's tourism to Taiwan in regards to employment opportunities of ordinary people and youth, development space of Taiwan travel agencies and related industries, protection and revitalization of local culture, stimulating community vitality, optimizing infrastructure development, and other positive effects, support of Taiwan residents for mainland' $\mathrm{s}$ tourism may increase. Efforts should be made to expand Taiwan residents' participation in order to further their understanding of and support to cross-strait economic and social developments.
\end{abstract}

Key words: relationship between resident and community; tourism support; influencing factors; community attachment; community concern 